\title{
A Cultural Study of Open-Palm Hand Gestures and their Prosodic Correlates
}

\author{
Gaëlle Ferré ${ }^{1}$, Amina Mettouchi ${ }^{2}$ \\ ${ }^{1}$ LLING, Nantes Université \& UMR 6310 CNRS, France \\ ${ }^{2}$ EPHE, PSL \& LLACAN UMR 8135 CNRS, France \\ gaelle.ferreduniv-nantes.fr, amina.mettouchi@ephe.psl.eu
}

\begin{abstract}
This paper presents an exploratory study of open-palm gestures with five recurring hand orientations and their use by storytellers speaking different languages, within distinct cultures - Metropolitan French, American English (US), and Kabyle Berber (Algeria). With the help of single (CA) and multiple correspondence analysis (MCA), we show that (a) storytellers of the three languages do not perform the same recurrent openpalm gesture orientations and (b) that they do not align their gestures with the same speech events: the speech prosody accompanying those gestures is different depending on the language of the storytellers. Overall, the study provides a preliminary picture of the various cultural stances adopted in the activity of storytelling.

Index Terms: open-palm hand gestures, prosody, Multiple Correlation Analysis (MCA), stance, storytelling, culture.
\end{abstract}

\section{Introduction}

Working on ordinary conversations, [1] found that some gestures are performed very frequently and that, contrary to the idiosyncratic gesture forms and movement types observed in representational gestures, the gestures that have a more pragmatic function show similarities in form and trajectory. He grouped them into what he calls 'gesture families': the G-family also called the 'finger bunch', the R-family (digits forming a circle or 'ring'), the open-hand prone family (palm down) and the open-hand supine family (palm up). Within each family, gestures acquire different functions depending on the type of movement and trajectory they are performed with, thus forming what other researchers have called 'recurrent gestures' [2] defined by $[3$, p. 1559] as "conventionalized to a certain degree, (...) culturally shared" and being "identified clearly within the stream of manual movements". The author also adds that "like emblems, recurrent gestures show a stable form meaning relation and can be distinguished from 'singular gestures' " (op. cit). Unlike emblems as they are described in $[4,5,6]$, however, those gestures are not autonomous from speech and can't be given a precise 'verbal translation'. Open-hand gestures have been the object of several studies, for instance [7] and [8] have worked on the different functions the PUOH (Palm-Up-Open-Hand) gesture can reveal in contexts of use, while $[9,10]$ described the palm-down and palm-away (also called the 'wall') gestures in the context of negation. [11] also gives a precise semiotic analysis of some of those recurrent gestures and of the pragmatic functions of various movement types. Apart from a few studies (e.g. [12]), the gestures were mainly described in the context of Western culture with a focus on the form-meaning relationship in context. Although, [10] does mention the performance of the palm-away gesture in China, his aim is not to point out any cultural difference. So far the gestures have been assumed to be more or less universal, with local variants in terms of gesture- speech relationships $[12,10]$. And indeed, all gesture forms are represented in corpora from different cultures or in different languages. Yet, are the various recurrent gestures used in the same proportion in different languages?

Investigating this question is the purpose of the present paper which compares open-hand gestures performed with five different orientations of the palm (palm up, palm down, palm away, palm on side and palm toward self) in three different languages, i.e. Metropolitan French, US American English and Kabyle (Berber). In video recordings of traditional tales told by six women, we observe both the proportion of each type of gesture performed by the storytellers, and the way the gestures are synchronized with speech. The prosodic and gestural observations lead us to conclude that the differences present in our corpus reveal a difference in stance-taking toward the activity of storytelling.

\section{Theoretical background on gesture form-meaning relations}

The palm-up open-hand gesture has most often been described as a recurrent gesture form "pointing to some object as if it were being 'presented' to the interlocutor as something that should be looked at or inspected" [1, p. 210]. In [8], it was noted that the PUOH gesture is used by speakers to add nuance to their speech through expression of some epistemic stance, i.e. uncertainty in most contexts.

The palm-on-side gesture has been described for twohanded gestures by $[11$, p. 71$]$ in what she calls the "vertical frame configuration' as metaphorically locating a defined discourse object in the physical space before the speaker, thus making it possible for the speaker to establish contrasts between different referents in speech. When performed with one hand, the same gesture rather has a dividing function between discourse items. The palm-on-side gesture, as well as the palm-up one, may also have a pointing function when oriented toward the interlocutor, and in this context, they have the interactive function described by [13], by which they include the interlocutor as sharing a viewpoint without having to mention this explicitly in speech [14].

The palm-away gesture was described by $[11$, p. 25] as expressing negation by simulating interruption or repulsion, avoidance, or retreat, but also p. 169 as a possible refusal to take responsibility for what is said or heard. [12] add that the gesture may also highlight topic changes or the insertion of additional information in speech.

The palm-down gesture is very close to the palm-away one insofar as it is also linked to negation. The gestures of the palm-down family "share the semantic theme of stopping or interrupting a line of action that is in progress. By 'line of action' we mean any project that someone might be engaged in, whether this involves physical action, communicative action 
(such as saying something), or mental activity, such as pursuing a train of thought or assuming a certain mental attitude toward something" [1, pp. 248-249]. When the two gestures are explicitly linked to some negation in speech, [10] notes that their synchronization with parts-of-speech indicates the scope of the grammatical negation (e.g. verb phrase, clause or whole sentence).

To our knowledge, the palm-toward-self gesture has not been studied in the literature but it can be considered as a deictic gesture [15] through which the speaker directly refers to him/herself. Finally, gestures also have a demarcative function according to [11] again, insofar as they align with different idea units.

\section{Data and methods}

\subsection{Corpus}

The corpus used for this study is composed of 6 videos featuring the telling of traditional tales by 6 women: 2 women were French, 2 were American and 2 were Kabyle (a Berber group living in Northern Algeria). While the videos in French and American English were downloaded from the internet ${ }^{1}$, the two Kabyle speakers were recorded in Algeria by the second author in the course of a long-term documentation project started in 1992. The 2 French tales last $10 \mathrm{~min}$ and $7 \mathrm{~min} 58 \mathrm{~s}$ respectively, the 2 American ones last 8 min $35 \mathrm{~s}$ and $12 \mathrm{~min} 48 \mathrm{~s}$, and the Kabyle videos last $13 \min 28 \mathrm{~s}$ and $9 \min 11 \mathrm{~s}$, so that the total duration of the tales is 1 hour $2 \mathrm{~min}$.

\subsection{Data}

Each video was transcribed manually in Praat [16] by one of the two authors. The text was transcribed verbatim for the videos in French and American English, using the Intonation Units (IUs) of the British tradition as a segmentation criterion [17]. We distinguished between minor (/) and major (//) intonation phrases, minor IUs being uttered with a continuation F0 contour whereas major ones were uttered with a conclusive contour. The first Kabyle video had already been transcribed into the IPA with the same IU segmentation in a previous project (CorpAfroAs ${ }^{2}$ ) and the second video was segmented and transcribed in the same way as the first one, for the purpose of this paper. Silent pauses and audible breath intakes were noted as well. Transcriptions (as well as the annotations described below) were systematically checked by the other author and discrepancies in coding were resolved through discussion.

In the video annotation tool Elan [18], we coded the different open-palm gestures in each video with the sound turned off, counting the palm orientations shown in Figure 1: palm away, palm down, palm on side, palm toward self and palm up. Gesture annotation was based on form only, without any distinction of gesture function in speech or movement type. We coded a total of 812 gestures and Table 1 below gives the detailed number of each gesture type performed by the speakers of the three languages as well as the sum per language and type.

\footnotetext{
${ }^{1}$ Catherine Zarcate: Le singe et le crocodile, "https://www.youtube. com/watch? $=$ IcUgBXaHN1U\&t=20s"; Muriel Bloch: Le schma doudou, "https://www.youtube.com/watch? $\mathrm{v}=\mathrm{dK} 0 \mathrm{p} 0 \mathrm{r} 7 \mathrm{hhBg} \& \mathrm{t}=25 \mathrm{~s}$ ", Yvonne Young: The elephant and the blind men, "https://www. youtube.com/watch? $v=$ sAcb8lajT2Q\&t=154s", Rachel Hedman: The magic ball, "https://www.youtube.com/watch?v=GbN1rLHeD84".

2"http://corpafroas.huma-num.fr"
}
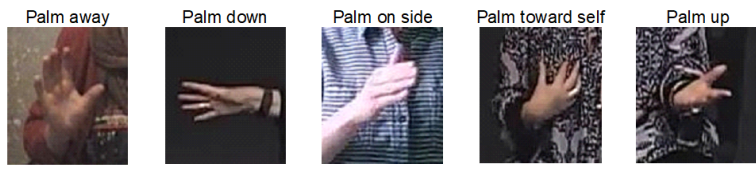

Figure 1: The five open-hand gestures in our study

Table 1: Number of open-palm gestures for each language

\begin{tabular}{rrrrr}
\hline & French & Kabyle & American & Sum \\
\hline palm away & 8 & 78 & 41 & 127 \\
palm down & 17 & 91 & 43 & 151 \\
palm on side & 30 & 69 & 99 & 198 \\
palm toward self & 16 & 8 & 14 & 38 \\
palm up & 161 & 66 & 71 & 298 \\
Sum & 232 & 312 & 268 & 812 \\
\hline
\end{tabular}

To be considered as an open-hand gesture, the speaker's hand(s) had to be open either in a tense or in a more relaxed way. The fingers could be held tight or spread. The gesture could be static or dynamic, but there had to be at least one stable image during its stroke for it to be included. Gestures in which the open-palm was obviously a secondary feature of the performance (like opening one hand to count on the fingers with the other one, or opening hands to clap) were not included. When the two hands showed a different palm orientation (most of the time this happened when one hand was held in a position stemming from a previous gesture while the other hand performed an open-palm), then the active hand determined the palm orientation of the gesture. Lastly, we also coded gesture apices (points of maximal extension) which served as reference alignment points for all the annotations (i.e., determined with which IU the gestures were synchronized). Other annotations were also coded in Elan after the speech tier was imported from Praat:

Turn Constructional Units (TCUs): groups of minor IUs that form a single piece of information as in the following example from our corpus: // Long long ago in India / there lived six blind men //.

Position in TCU: TCUs were divided into 3 temporally equal segments and we coded gesture apices as occurring either at the beginning, in the middle or at the end of TCUs irrespective of the type of IU (minor or major) they co-occurred with.

IU type: we noted whether the IUs preceding, co-occurring with, or following gesture apices were minor or major ones in three separate tracks.

Pauses: pause type before the co-occurring IU or after it were coded as silence, breathing or complex when the two were combined.

The rest of the annotations were made with Praat and reference points were gesture apices. These included :

Intonation contour of the IU containing the gesture apex, as well as the contours of the preceding and following ones.

Pitch reset at the beginning of the IU that co-occurred with gesture apex or at the beginning of the following IU.

Presence or absence of a focal accent (a marked prosodic prominence which can have a contrastive or emphatic value, mostly expressed by extra lengthening of a syllable or syllable onset and/or higher pitch). 


\subsection{Research questions and statistical analysis}

The aim of our study was to investigate whether types of openpalm hand gestures vary across different languages, and how they align with the prosodic features of the accompanying speech. In order to answer those questions, we conducted a simple and a multiple correspondence analysis (CA and MCA, [19]) with the R statistical software [20] using the packages FactoMineR and factoextra for multivariate analysis. CA is used to analyze frequencies formed by two categorical data, while MCA is used to detect and represent underlying patterns in categorical data with several variables. The interpretation of MCA is based on the proximity of points on a two-dimensional map (the closer the points on the graph, the higher the correlations between the two variable values) and "is part of a family of descriptive methods (e.g., clustering, factor analysis, and principal component analysis (PCA)) that reveal patterning in complex datasets" [21].

\section{Results}

The graph in Figure 2 represents the results of the correspondence analysis between language and use of open-palm hand gestures. It reveals a strong correspondence between Kabyle and the use of both palm-away and palm-down gestures, while the American tellers rather use palm-on-side gestures and the French use palm-up ones. The palm-toward-self hand orientation is not significantly used by any cultural group. Although the French performed more of those, there were few occurrences of this gesture type in the whole corpus as shown in Table 1 , which makes it stand out from other gestures. The graph also reveals that Kabyle storytellers's profile is distant both from the French, who stand at the opposite side of the 1st dimension, and from the Americans who also stand on the opposite side of the 2nd dimension, although Kabyle storytellers are closer in their gesture performance to the Americans (with coordinates ranging from -0.2 for KA to 0.3 for US tellers on dimension 2) than to the French (with coordinates ranging from -0.4 for KA to 0.75 for FR tellers on dimension 1 ).

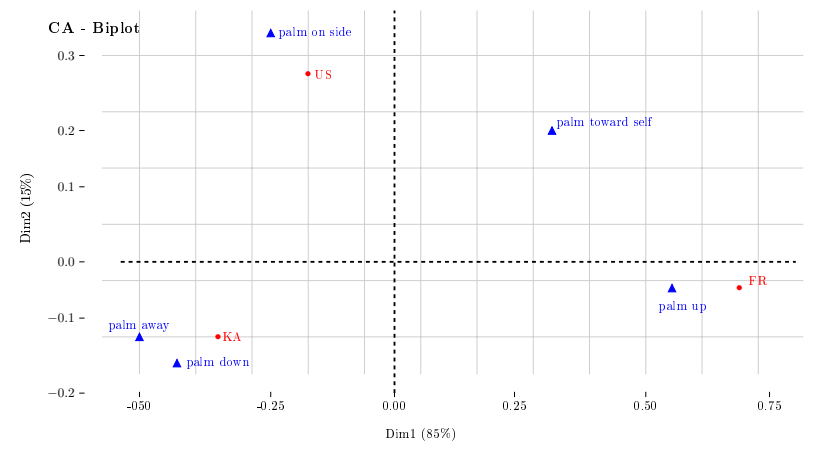

Figure 2: Biplot of the correspondence analysis between gesture orientation (in blue) and language (in red)

Figure 3 presents the contributions of the different variables we coded to the two dimensions of the graph visible in Figure 4. Any variable that does not reach the red dashed line does not contribute to the dimension (i.e. does not show any correlation with language or hand orientation). "For a given component, a variable with a contribution larger than this cutoff could be considered as important in contributing to the component" $[19$, p. 26]. The variables that account for the highest
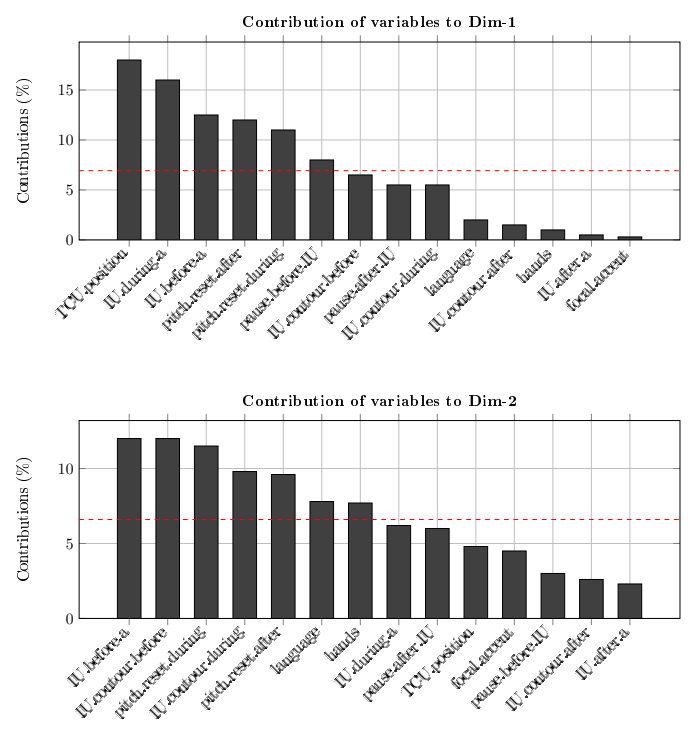

Figure 3: Contributions of variables to dimensions 1 and 2.

variance are the position of gesture apex in the TCU and the type of co-occurring IU (minor or major) for dimension 1, and the type and intonation contour for the IU located before gesture apex as well as the presence/absence of pitch reset in the co-occurring IU for dimension 2. The type (minor vs major) and intonation contour of the IUs that follow gesture apices, the presence/absence of a pause after their co-occurring IUs as well as the presence/absence of a focal accent on the co-occurring IUs do not contribute to any of the two dimensions. These variables were therefore not taken into account in the rest of the analysis.

The graph in Figure 4 below presents the storytellers of the three languages in three different squares of the two-dimension plot issued by the Multiple Correspondence Analysis (MCA). The prosodic features in each square are the ones which correlate the most with each language and gesture palm orientation. The figure presents our results for the 6 speakers involved in the study, with negligible variation between speakers inside each language group, despite variation in the contents of the stories.

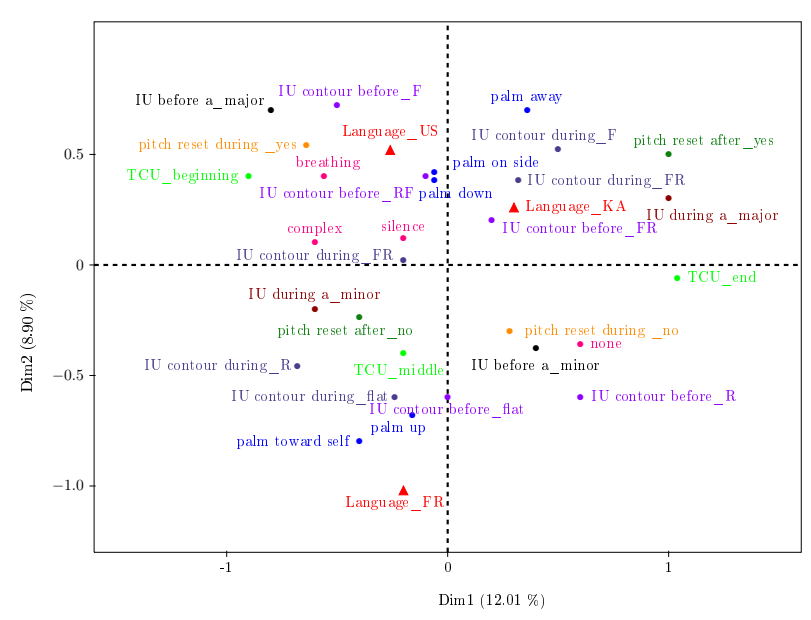

Figure 4: Multiple Correspondence Analysis 
The French storytellers are represented in the bottom left square of the plot. Their most frequent gesture orientations are the palm-toward-self and palm-up gestures, which are generally performed in the middle of the TCU with typically no pitch reset after the IU they are aligned with. The co-occurring IU is a minor one with a flat or rising F0 contour while the contour of the preceding IU is also flat.

The Kabyle storytellers are shown in the upper right square of the plot. Their preferred hand orientation is the palmaway gesture, but they also perform quite a few palm-down hand gestures (an orientation shared with the Americans). They align the gestures preferentially with the end of the TCU (although this correlation is weak), and with a pitch reset on the following IU. The contour of the major IUs they accompany is either a rise fall $(\mathrm{RF})$ or a simple fall $(\mathrm{F})$, while the preceding IU has a fall rise (FR) contour.

The American storytellers are represented in the upper left square of the plot. Their preferred gesture orientation is the palm-on-side, although, as stated above, they also perform quite a few palm-down gestures. Their gestures are aligned with the beginning of the TCU and the IUs they accompany are typically preceded by a pause (especially audible breathing but other pause types are present as well). The contour of these IUs is most often a fall rise (FR) and they are uttered with a pitch reset, while the contour of the preceding major IUs is a rise fall or a fall.

\section{Discussion and conclusions}

What the results presented in the previous section shows is that while the five gesture forms under study are all performed by the speakers of the three languages in their tales, they are not recurrent to the same degree. The Americans show a strong preference for the palm-on-side gesture, performed at the beginning of the TCU, in a minor IU preceded by a pause and uttered with a falling rising tone, that indicates more is to come. The function of this gesture as presented in section 2 is to delimit a segment of speech from the preceding information unit and adding new narrative events. The speakers here do not express any particular stance on the content of their speech.

On the contrary, the French preferentially perform the palm-up gesture in the middle of the TCU, in synchronization with a minor IU uttered with a flat or rising contour. This also indicates that the TCU is still incomplete at the moment the gesture is performed, but the fact that the gesture apex is aligned with the middle of the TCU (typically corresponding to the rhematic/new part of the information) shows that it is this part of the information that is highlighted by the gesture, although no focal accent is present. What the gesture adds to the speech content is therefore not so much some additional prominence to speech items, but the presentation of the information to the listener for inspection. The fact that the French also use more palm-toward-self gestures - although in a much smaller proportion - also shows that the telling activity is conceived as sharing a tale between audience and storyteller, and not simply focusing on the succession of events.

The Kabyle storytellers perform the palm-away and palmdown gestures aligned with the end of TCUs, in major IUs uttered with a rise fall or a fall and followed by a pitch reset Contrary to the findings described in section 2 which regularly associate those two gestures with negation, they do not co-occur with negative clauses in the Kabyle tales presented here. Their co-occurrence with TCU endings and (rise-)fall contours indicate that they are associated to some degree of finality, either in stance, or in narrative organization. A more precise study of the pragmatic and semantic contents of the IUs they co-occur with is under way, but their general frequency, compared to the French and the Americans' gesture profile, evokes a culturespecific stance towards storytelling not found in the other two languages, despite the fact that all six stories are broadly comparable (third-person fictional narratives). Indeed, while the Americans and French share a common culture based on oral transposition of written texts in a way that favors individual style and performance, and allows for considerable variation in contents, the Kabyle storytellers are non-literate speakers performing within a still vibrant oral tradition in which it is very important that the tales be transmitted in a form that is highly coded, and much less individual than in the Western tradition. In that tradition, tales are introduced by a formula that underlines the role of the storyteller as a vessel through which the tale is transmitted: "May my tale be beautiful, may it come out like a woven ribbon". The rhythmical delivery, the set formulas, and a series of prosodic forms (such as complex pauses involving audible breath intakes as shown in [22]) work like a musical piece, thus sustaining memorization and performance, as well as the passing-on of elements of culture. Expanding on the functions so far found in the literature for the palm-away gesture, all involving some kind of distanciation, or 'refusal to take responsibility' (section 2 and [11, p. 169]), that gesture (and to a certain extent the palm-down gesture) can therefore be seen as ways of expressing some form of non-individual stance-taking. When those gestures do not co-occur with a character's individual dismissal or rejection in reported dialogue, their function is in most cases to express that the storytellers are not the individual authors of those tales and that they are passing on the stories following a tradition that goes beyond their individual performance.

This study thus showed different cultural uses of recurrent gestures and opens up an avenue of research in cultural gesture studies. For instance, it would be interesting to compare the use of the gesture palm orientations by speakers of more languages and cultures in various speech genres, but even when remaining in the realm of traditional storytelling in the same languages, it would still be interesting to compare the performance of speakers of different varieties. Do Canadian French and British speakers, for instance, perform the same types of gestures as people speaking the same language, or have they adopted the gesture habits of their geographical neighbors? Are the gestures performed by our two Kabyle women specific to the particular language in which the tales are delivered or do they share their recurrent gestures with people speaking different languages in other oral cultures? Lastly, the study conducted here involved six women and one may wonder if men perform the same types of gestures as well or if the gestures are gendered.

\section{Acknowledgments}

This research received no specific grant from any funding agency in the public, commercial or nonprofit sectors. We are therefore especially grateful to the storytellers for either sharing their tales on the internet or for accepting to be recorded and thereby contributing to linguistic research. We are also grateful to anonymous reviewers for their helpful comments on a previous version of this paper. 


\section{References}

[1] A. Kendon, Gesture. Visible Action as Utterance. Cambridge: Cambridge University Press, 2004.

[2] C. Müller, "How Recurrent Gestures Mean," Gesture, vol. 16, no. 2, pp. 277-304, 2018.

[3] S. Ladewig, "Recurrent Gestures," in Body-Language - Commu nication, C. Müller, A. Cienki, E. Fricke, S. H. Ladewig, D. McNeill, and S. Teßendorf, Eds. Berlin, Boston: Mouton de Gruyter, 2014, pp. 1558-1574.

[4] P. Ekman and W. Friesen, "Hand movements," Journal of Communication, vol. 22, pp. 353-374, 1972.

[5] L. Payrató, "A pragmatic view on autonomous gestures: A firs repertoire of Catalan emblems," Journal of Pragmatics, vol. 20, no. 3, pp. 193-216, 1993.

[6] S. Teßendorf, "Emblems, quotable gestures, or conventionalized body movements," in Body - Language - Communication, C. Müller, A. Cienki, E. Fricke, S. H. Ladewig, D. McNeill, an S. Teßendorf, Eds. Berlin, Boston: Mouton de Gruyter, 2013, pp. 82-100.

[7] C. Müller, "Forms and uses of the palm up open hand: A case of a gesture family?" in The semantics and pragmatics of everyday gestures, Müller, C. and Posner, R., Ed. Berlin: Weidler, 2004, pp. 233-256.

[8] G. Ferré, "Functions of Three Open-Palm Hand Gestures," Multimodal Communication, vol. 1(1), pp. 5-20, 2011.

[9] S. Harrison, "The Expression of Negation through Grammar and Gesture," in Studies in Language and Cognition, J. Zlatev, M. Andrén, and C. Lundmark, Eds. Cambridge: Cambridge Scholars Press, 2009, pp. 405-419.

[10] - The Impulse to Gesture: Where Language, Minds, and Bodies Intersect. Cambridge: Cambridge University Press, 2018.

[11] G. Calbris, Elements of meaning in gesture, ser. Gesture Studies. Amsterdam, Philadelphia: John Benjamins, 2011.

[12] J. Bressem, N. Stein, and C. Wegener, "Structuring and highlighting speech - Discursive functions of holding away gestures in Savosavo," in GESPIN 4, G. Ferré and M. Tutton, Eds., Nantes, France, 2015, pp. 49-54.

[13] J. Beavin Bavelas, "Nonverbal Aspects of Fluency," in Perspectives on Fluency, H. Riggenbach, Ed. Ann Arbor: University of Michigan Press, 2000, pp. 91-101.

[14] T. McGowan, "Abstract Deictic Gestures-in-Interaction: A Barometer of Intersubjective Knowledge Development in SmallGroup Discussion," Working Papers in Educational Linguistics, vol. 25(2), pp. 55-79, 2010.

[15] D. McNeill, Gesture and Thought. Chicago and London: University of Chicago Press, 2005.

[16] P. Boersma and D. Weenink, "Praat: Doing Phonetics by Computer (Version 5.1.05) [Computer program]," 2009.

[17] J. C. Wells, English Intonation. An Introduction. Cambridge: Cambridge University Press, 2006.

[18] H. Sloetjes and P. Wittenburg, "Annotation by Category - ELAN and ISO DCR," in Proceedings of the 6th International Conference on Language Resources and Evaluation (LREC 2008), Marrakech, Maroc, 2008.

[19] A. Kassambara, Practical Guide to Principle Component Methods in R. www.sthda.com/english/: Statistical Tools For HighThroughput Data Analysis (STHDA), 2017.

[20] R Core Team, "A Language and Environment for Statistical Computing. R Foundation for Statistical Computing. computer program: [http://www.r-project.org]," 2012.

[21] P. S. Costa, N. C. Santos, P. Cunha, J. Cotter, and N. Sousa, "The Use of Multiple Correspondence Analysis to Explore Associations between Categories of Qualitative Variables in Healthy Ageing," Journal of Aging Research, vol. 2013, p. 12, 2013.

[22] A. Mettouchi, "Audible breath intakes in monologues," Journal of Speech Sciences, vol. 7, no. 2, pp. 93-106, 2018. 